\title{
Envisioning Mobile Information Services: Combining User- and Technology-Centered Design
}

\author{
Jesper Kjeldskov and Steve Howard \\ Department of Information Systems \\ The University of Melbourne \\ Parkville, Victoria 3010, Australia \\ jesper@cs.auc.dk, showard@unimelb.edu.au
}

\begin{abstract}
We provide a meta-commentary on two approaches used for designing context-dependent mobile devices. On the basis of a 'user-centered' approach, consisting of interviews, observation of current practice and enactment of future scenarios in context, a number of non-functional design sketches were developed. While these sketches reflected a rich understanding of current work practices, they were little more than abstract speculations about future practice; lacking in detail on usability and feasibility, and being largely reactive to current problem situations. Conducted in parallel, the technology-centered study informed the design and implementation of a mature functional prototype. This facilitated a comprehensive usability evaluation revealing a series of technical challenges and problems related to mobile use. Though the technology-centered approach provided detailed input for refining the prototype, and an initial provocative break with current practice, it was less useful in supplying further original alternatives; post-evaluation, the design discussion was largely reactive to the current prototype. In concert, the two approaches complement each other well; the user-centered approach grounding design in current practice, in all its contextual complexity, and the technology-centered approach providing a counterpoint in technically detailed expressions of future possibilities.
\end{abstract}

\section{Introduction}

Mobile, pervasive and ubiquitous computing constitutes a challenge for humancomputer interaction design. Like other emerging technologies, these technologies are characterized by being different from traditional desktop computers in their physical appearance and the contexts in which they are used. Consequently, they often imply interaction dissimilar from how computers are usually operated and are used for purposes beyond office and home computing. Many traditional approaches to HCI design are evolutionary and based on iterations of analysis, design, implementation and testing. These methods have proven valuable in relation to the development of computer systems with high usability based on well-known and widely adopted technologies such as desktop workstations and the Internet. In the light of emerging technologies, however, interaction design research has been stimulated into the development and use of design approaches that are less analytic and more creative. These approaches supplement traditional HCI methods by introducing an explicit focus on 
envisioning future technology and its use - so called 'blue-sky' research. The fundamental assumption is that just like the introduction of personal computing and computer networks have led to huge and unexpected changes in the work and leisure activities of many people, so will mobile, pervasive and ubiquitous computing in the decades to come. As the potential use of these technologies is, however, still unknown, researchers must develop new concepts and ideas for future technology: what it may look like, what it may be used for, and how people may interact with it.

HCI research promotes two overall approaches to concept design for emerging technologies: 1) experiments driven by the opportunities offered by new technology and 2) user-centered design driven by field studies. While a recent literature survey [7] shows a tendency towards mobile HCI being driven by technology- rather than user-centered approaches, growing attention has lately been brought to concept development methods focusing on present and envisioned future user activities. Much of this research has its roots in the Participatory Design tradition [2], and is motivated by the observation that "we can make amazing things, technically, but are often at a loss to understand what to make", as stated by Vygotsky [12]. In response to this, new methods are introducing techniques from theatre, such as actors, props and roleplaying to HCI research. Sato and Salvador [11] suggests a focus group approach modified to involve actors and an active audience acting-out use of existing and nonexisting product concepts. The benefits from this approach are that the theatre approach creates a strong context with focus on interaction as well as an improvisational space for the participating users. However, it is also noted that it can be difficult for users to be creative and articulate on non-existing products in fictive use scenarios. Inspired by this work, Howard et al. [3] have experimented with the use of 'endowed props' providing the design team with a means of directing the discourse between science- and plausible-fiction. Extending this line of thought, Iacucci et al. [5] presents techniques where enacting the use of future technology is a part of a board game or takes place in the real world (the SPES technique), thus restricting discourse by means of a game master or the constraints of real life activity.

These and related techniques indicate a plausible approach to interaction design for emerging technologies. However, they are to a large extent disconnected from real technology. While on one side this frees designers from the technological constraints of the present, it also inhibits the exploration of new technological potentials and may blind insight into limitations of present and emerging technology. Hence, Rogers et al. [10] promotes that instead of choosing between a user- and a technology-centered approach, blue-sky research should explicitly involve both playful visions of technology in their social context and innovation through 'technology inspiration'.

On the basis of this discussion, this paper presents a research project in which a user- and a technology-centered approach were combined for envisioning design of mobile information services for the same problem domain. The first section describes the motivation and methodological design of the project. Following this, the details of the user- and the technology-centered approaches are described and discussed. This includes a description of the empirical methods used, the designs produced and the evaluations conducted. Finally, the interplay between the two approaches is discussed and directions for further research are outlined. 


\section{Supporting Use of Public Transport by Mobile Computing}

Between October 2002 and April 2003 we conducted a research project focusing on the potential use of context-aware mobile computing for supporting the use of public transportation in Melbourne, Australia. The project was motivated by discussions among consultants and sales staff of a large IT company about alternatives to the use of cars for traveling in city meetings with clients. In large cities where traffic is often very dense, traveling by car can be highly time-consuming, necessitating much planning. Using Melbourne's tram-based public transport would not only be more environmental, but might also be more effective if supported by a mobile information service providing travelers with relevant information at the right time and place. Over the course of six months, a team consisting of three senior researchers, five $\mathrm{Ph} . \mathrm{D}$. students, two research assistants and an industrial interaction designer worked on different parts of the project. The first author of this paper managed the project and participated in the majority of activities. The researchers were all involved in HCI design for mobile computing but had different specific individual research interests ranging from ethnographic user-studies to prototype development and usability evaluation. On the basis of the different points of interest, the project consisted of two parallel tracks of research: a user- and a technology-centered track (figure 1).

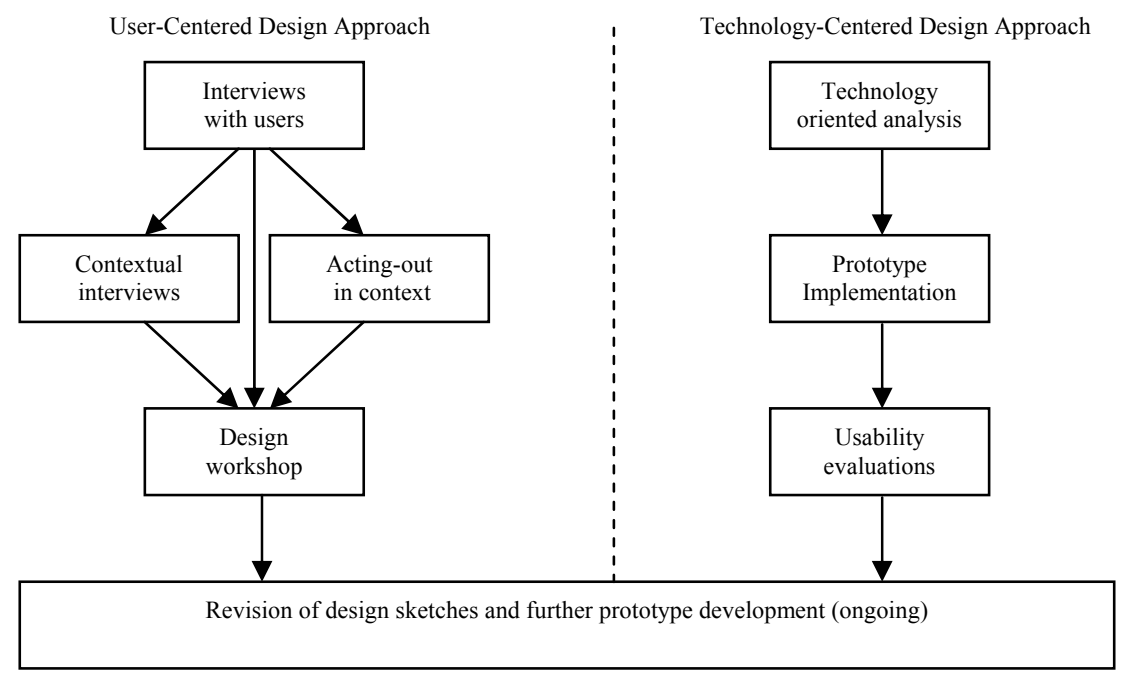

Fig. 1. Combining user- (left) and technology- (right) centered approaches to interaction design

The user-centered track grew out of the initiating discussions with the IT professionals. The technology-centered track grew out of an already ongoing activity to develop a mobile route-planning service for the tram system of Melbourne. The researchers in each track remained mutually unaware of each other's activities for the first 3 months of the project. Thus the user studies were conducted without any knowledge of the prototype development and the design of the first experimental prototype was not informed by the empirical user studies. Following the design workshop, researchers joined across the tracks and conducted a usability evaluation of the prototype. Finally, 
the outcomes from the two tracks of research were merged. The following sections describe the activities in the two parallel tracks in detail.

\section{The User-Centered Approach}

Based on the discussion above, we designed a user study to investigate into the current and possible future work practices of our nomadic users. In order to construct a rich understanding of travel and work practices, and building on project members' experience with techniques for acting-out future scenarios from previous research [3] we combined three empirical methods: 1) Semi-structured interviews with users (at the office), 2) Contextual interviews and observation of current practice (driving cars in city), and 3) Acting-out future scenarios in context (on board trams in city)

These methods all involved users talking about their perceptions, activities and needs. While the semi-structured interviews were separated from context, the contextual interviews and acting-out sessions took place while the users were carrying out real work activities. During the contextual interviews and acting-out sessions, we observed current practice, including the use of different artifacts in relation to the work activities being carried out. In the acting-out sessions we furthermore provided a series of non-functional props to assist the users' imagination. The three methods and a summary of their primary outcomes are described below.

\subsection{Interviews}

To establish the context, four semi-structured interviews were conducted with the IT professionals, who frequently traveled for work activities in the city. The interviews focused on the users' perceptions of pros and cons of traveling by car and tram. Each interview was conducted by a pair of researchers and lasted 30-60 minutes.

It was clear from the interviews that the users were willing to use public transport when attending meetings in the city, but that the use of the local tram system had a number of limitations. Firstly, uncertainties were related to route planning: which routes to take, when to change trams, how many changes would be needed, would there be a wait at each change, how close was the tram stop to the desired destination...? Often users would tend towards their car, or a taxi, if tram changes were needed, or if the journey was to an unfamiliar destination. Being able to predict the time of arrival of the tram rather precisely was seen as critical. The interviewees attempted to avoid being either late or too early (thus wasting time for their clients, or themselves respectively). To assist in timely arrival at appointments the interviewees needed to know exactly when to leave their office in order to arrive at their meeting just at the right time. When using the car this estimation was reportedly done based on knowledge about normal traffic conditions in the city and was not very precise. Uncertainty about combining routes, trams not running on schedule, finding the nearest stop and having to walk all constituted barriers to tram use. 


\subsection{Contextual Interviews and Observation of Current Practice}

Following the interviews, the next phase consisted of observing and enquiring into current practice (figure 2). Here we were trying to understand both hurdles and enablers to car and tram travel. The observations were conducted by shadowing the users during travel from their offices to meetings in the city. One researcher asked questions about pros and cons of using trams while another video taped the session.

From the observations we learned that, although driving to meetings in the city could be rather time consuming, the car was seen as flexi-

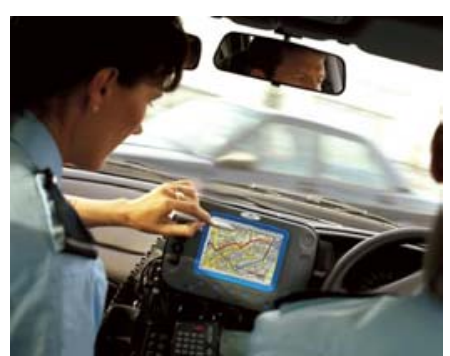

Fig. 2. Contextual interview and observation of current practice ble and provided a useful 'office on the move', a semi-private space for limited work activities. Thus time spent in the car was not necessarily 'wasted' time but often used for preparing the upcoming meeting or coordinating other activities over the phone.

\subsection{Acting-Out in Context}

The final phase of our field study consisted of a number of sessions in which our nomadic users acted-out future scenarios of using mobile information technology to support travel by tram (figure 3). The acting-out approach was adapted from previous research by project participants [3].

When acting-out a future scenario, people are asked to envision and enact situations involving future use of technology, based on an overall frame of context and supported by simple props which are attributed the desired functionality. For

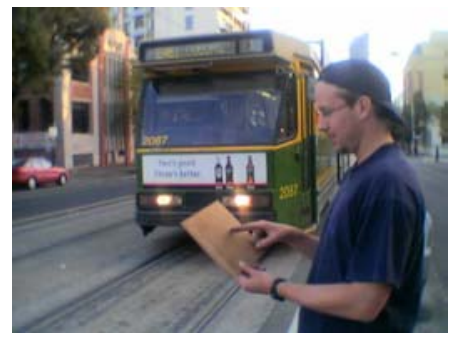

Fig. 3. Acting-out in context the present project, the original approach was modified in a number of ways, resembling aspects of the SPES technique [5]. First, real users instead of actors did the acting-out. Secondly, the acting-out was done in context: using the trams for attending a real meeting in the city instead of performing fictive tasks in a studio. Two researchers facilitated the sessions and took notes; a third recorded the sessions on video.

From the acting-out sessions we learned a lot about information needs and desires and how these varied across different situations and locations. Also criteria for assessing the appropriateness of different form factors were revealed, for example the need for privacy when working in a public space. Specifically, the acting-out in context sessions revealed that before catching a tram into the city, estimation of travel time was essential to determine when to go. On the tram, the primary information needed was when and where to get off and what to do next. In addition, users envisioned that they could prepare a meeting, browse the Internet or listen to music. One user also envisioned that the device would automatically buy an electronic ticket from the tram. 


\subsection{Design Workshop}

Following the field studies, we conducted a one-day design workshop. The purpose of this was to recap and discuss the outcome of the empirical user studies and to produce a number of ideas for mobile systems supporting current and/or envisioned future practice. The workshop involved three senior researchers, three Ph.D. students, two research assistants and one industrial interaction designer. The latter managed the workshop in collaboration with the first author. First the three teams of researchers who had conducted the interviews, contextual interviews and acting-out sessions presented their findings. This was followed by a one-hour joint discussion. The participants were then divided into three groups, mixed so that they did not resemble the teams who did the user studies. The groups then spent $2 \frac{1}{2}$ hour on producing a design sketch of their own choice. No restriction was put on their focus, but the design had to address issues identified in the user studies. Following this, each group presented their design sketches using whiteboards and paper drawings. The workshop resulted in four design concepts. The ideas were highly creative and diverse: from a foldable mobile office to an MP3 player with voice-based route-planning, capabilities for presenting PowerPoint slides and wireless connectivity to stationary $\mathrm{I} / \mathrm{O}$ devices at the customer's site. The envisioned concepts all addressed different aspects and challenges of the mobile work activities studied and were informed by both input from the interviews, the contextual interviews and the sessions of acting-out. Due to limited resources and time, only one of the concepts, described in detail below, was developed further.

\subsection{TramMate}

TramMate supports the use of public transportation by means of a context-aware mobile calendar application. On the basis of the field study, the basic idea of TramMate is to 1) relate traveling information directly to appointments, 2) provide route planning for the tram system based on current location 3) alert when it is time to depart, and 4) provide easy access to travel time, walking distance and number of route changes. Elaborating on ideas of context awareness [1] and indexicality [8], the interface of TramMate is indexed to contextual factors such as time, location, and activity [6].
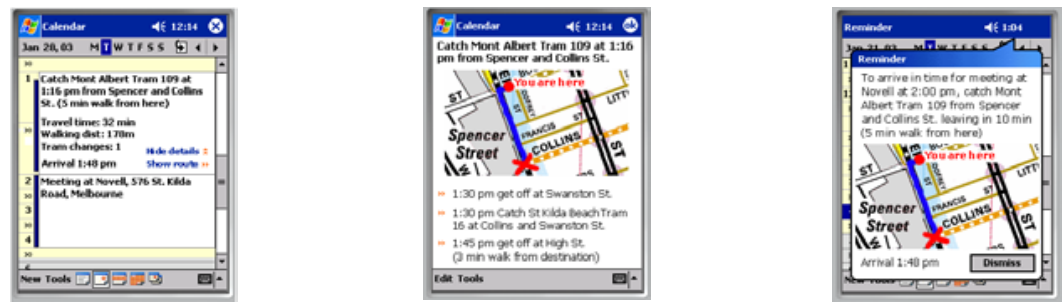

Fig. 4. Refined design sketches for TramMate 
The rationale behind TramMate is not to impose too much additional complexity on the user. Accomplishing this, an extended electronic calendar provides dynamic route planning information related to the user's schedule for the day. TramMate thus requires very little additional interaction. When a new appointment is made, the user is asked to specify the physical location of it, following which TramMate schedules a time slot for getting there. When an appointment is coming up, this timeslot adjusts itself in accordance with the location of the user and the estimated time needed to get there based on real time information about the public transport system (figure 4 left). Apart from specifying the first step of the route plan, the calendar view also provides additional details on the suggested route: estimated travel time, required walking distance and the number of route changes. During traveling, the TramMate timeslot continuously updates itself with information about the next leg of the route. From the calendar view, the user also has access to a screen providing his current location on a map with directions to the nearest tram stop. This screen also outlines the full route (figure 4 center). Based on the time required to walk from the user's current location to the first tram stop, TramMate notifies when it is time to leave in order to make the appointment. The reminder contains simple information about the appointment, what tram to catch, how soon it leaves, where it leaves from and how to get there (figure 4 right). On the tram, TramMate notifies when to get off and what next step to take. Arriving at the destination, a map provides the location of the appointment.

\section{The Technology-Centered Approach}

In parallel with the user-centered approach, researchers at the University of Melbourne's Department of Geomatics conducted a technology-centered track of research with the purpose of developing a functional prototype for Melbourne's tram system. The prototype addressed the same overall use-situation involving nomadic workers in the city that motivated the user study, but was not influenced by the findings in this track of research. Instead, it was driven by a desire to explore the potentials for providing location-based route planning on GPS and GPRS enabled handheld computers.

The prototype had a number of differences from and similarities to the TramMate concept. The prototype was similar in providing route-planning facilities for the tram system based on the user's current location as a mix of textual instructions and annotated maps. However, it provided a fundamentally different user interface, requiring the user to actively look up travel information rather than relating it to planned activities, and did not alert the user before or while in transit. Also, the prototype did not automatically reflect contextual changes such as the user changing location. This was only taken into account when the user actively looked up timetable information.

\subsection{Usability Evaluations}

Whereas the design sketches produced in the workshop were non-functional, the prototype enabled us to study the use of real technology. We conducted two usability evaluations of the prototype: in the field and in a usability lab, involving ten subjects. 
The evaluations were identical in terms of tasks and the profiles of test subjects. The subjects had to complete three realistic tasks involving route planning while traveling to appointments in the city derived from the user studies with IT professionals.

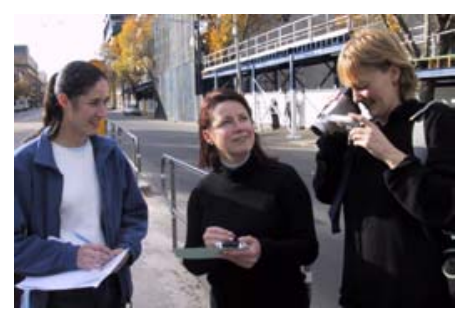

Fig. 5. Field evaluation

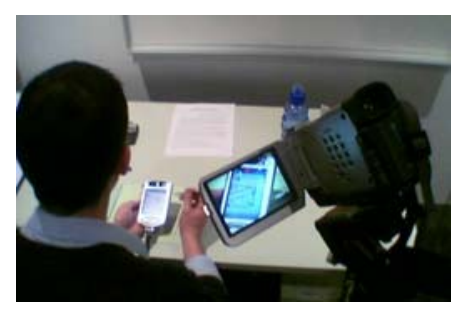

Fig. 6. Laboratory evaluation

The field evaluation focused on use in realistic settings. The test subjects had to lookup necessary information on the device according to the tasks and then perform the tasks "for real" (e.g. catching a tram to a specific destination). The prototype accessed live timetable information on Internet but GPS positioning had to be simulated. One researcher encouraged thinking-aloud, one took notes and one recorded the evaluation on video (figure 5). In the laboratory evaluation, the subjects were seated at a desk, with the mobile device in their hand (figure 6). To ensure a good video recording, the subject held the device within a limited area indicated on the table. Two researchers observed through a one-way mirror and took notes.

Studying the use of real technology revealed a number of problems concerning the design of context-aware mobile information services for supporting the use of the tram system. Overall, two critical and nine serious themes of usability problems were revealed [9]. One of the major problems identified concerned the relation between information in the system and in the world. During use, the context changed constantly; the users moved, the trams moved, time went by etc. While this was to some extent reflected in the system, the granularity of this relation was not sufficient. Consequently, the information on the screen would often be often 'out of sync' with the real world. Another major problem concerned the graphical design of maps. All users wanted to use the maps a lot. However, the maps turned out to have three significant limitations. Firstly, the level of detail was generally not appropriate. Either the screen would be cluttered or it would provide second to no information. Secondly, the maps lacked annotation of key information such as landmarks and precise indication of the user's location, route and destination. Thirdly, the users had serious problems relating the orientation of the map to the real world and would frequently rotate the device. As a consequence, screen text was often viewed upside down.

Other problems identified included difficulties entering data while being mobile, missing vital information due to lack of visual attention, and lack of functional transparency when information on the screen changed to reflect contextual changes. 


\section{Discussion and Conclusions}

It is interesting to compare the outcomes of the two parallel tracks of R\&D. The usercentered design approach facilitated the development of design concepts representing considerable insight into present work practices, and some speculations about the future not envisioned by the prototype designers. However, the usability and feasibility of these concepts were unclear. The technology-centered approach revealed specific technical challenges and problems related to the design and use of context-aware mobile information services not considered in the user-studies. However, aside from the initial visionary prototype, the technology-centered approach largely provided input for refining the implemented design rather than informing original alternatives, thus endorsing a trial-and-error approach [7].
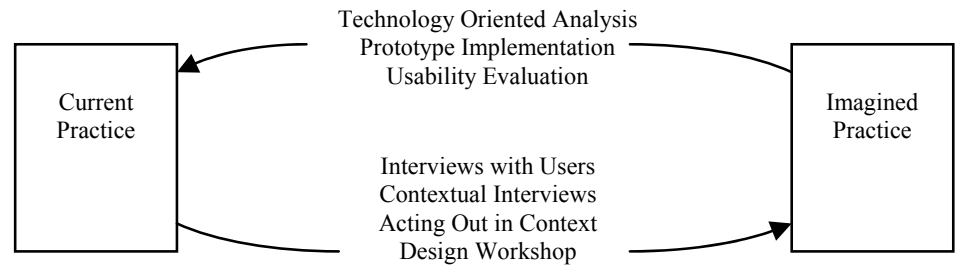

Fig. 7. Interleaving user- (bottom) and technology- (top) centered interaction design

With careful interleaving however, the two approaches complemented each other in the revision of design sketches and development of further prototypes by providing both playful visions and technology inspiration based on a mix of contextual richness and technical detail. Fig. 7 illustrates this interleaving, describing design as the exploration of the relation between an understanding of current practice (acquired in this case through the interviews and contextual interviews) and speculations about future practice (expressed as the technology prototype, and explored in the evaluations).

In the technology-centered approach, usability evaluation is used as a way of examining the impact, on current practice, of technology prototypes. Design proceeds by 'imposing' the design on the current situation of use during the evaluations, thereby exploring the design's impact on that use. In the user-centered approach, design moves from an understanding of current practice to imagined future use, thus driving design with user needs. Employing both a user- and technology-centred approach, results in a design process that attempts the co-evolution of an understanding of both current and future use, delivering innovative technology that is grounded in use [4].

The technology-centered approach informed the revision of the TramMate concept by identifying those envisioned design solutions that were challenging to implement or simply not feasible. Some examples may illustrate the interplay between the approaches. The critical user need (clear from the user-centered study) of maintaining a close relation between the system and the real world was not to be satisfied by the granularity of current GPS technology (clear from the evaluations of the technical 
prototype); especially on trams, other means of positioning would be required. Further, studying use of real technology showed that requirements for the user to devote their full attention to the device were excessive, as envisaged in the acting-out sessions. Also, an unexpected amount of effort would have to be put into the design of the maps; including pointers to landmarks, reflecting the orientation of the user etc. Finally, additional functionality was suggested such as manual timetable lookup. The user-centered approach assisted in the revision of the prototype by indicating and prioritizing information needs, outlining realistic use scenarios and proposing a novel interaction design concept integrating the system into another application. In short, the user-studies taught us a lot about what the system should do, while the evaluations of the prototype provided valuable insight into what could be done and feedback on the usability of what a specific design solution did do.

The presented project points towards many opportunities for future research. On a system design level, the revised design concepts should be implemented and evaluated. On the methodological level, it would, among others, be interesting to enquire into the potentials of managing discourse in blue-sky research envisioning future design concepts by introducing functional prototypes into acting-out sessions.

\section{Acknowledgements}

Thanks to colleagues on the TramMate project: Jennie Carroll, John Murphy, Jeni Paay and Frank Vetere (user-centered component), Connor Graham, Sonja Pedell and Jessica Davies (technology-centered approach). Thanks also go to professionals who participated in the user studies and to the usability evaluation test subjects.

\section{References}

1. Cheverst K., Davies N., Mitchell K., Friday A. and Efstratio C. (2000) Developing a Context-Aware Electronic Tourist Guide: Some Issues and Experiences. Proceedings of CHI'00, The Netherlands, ACM

2. Greenbaum J. and Kyng M. (eds.) (1991) Design at Work: Cooperative Design of Computer Systems. London, Lawrence Erlbaum Associates

3. Howard S., Carroll Jennie., Murphy J. and Peck J. (2002) Endowed props in scenario based design. Proceedings of NordiCHI'02, Denmark, ACM

4. Howard, S., Carroll, Jennie., Murphy, J., and Peck, J. 2002, Managing Innovation in Scenario Based Design. In Proceedings of Human Factors 2002, Melbourne.

5. Iacucci, G., Kuutti, K. and Ranta, M. (2000) On the Move with a Magic Thing: Role Playing in Concept Design of Mobile Services and Devices. Proceedings of DIS'00, ACM

6. Kjeldskov J., Howard S., Murphy J., Carroll Jennie., Vetere F. and Graham C. (2003) Designing TramMate - a context aware mobile system supporting use of public transportation. Proceedings of DUX'03, San Francisco, CA, ACM

7. Kjeldskov J. and Graham C. (2003) A Review of MobileHCI Research Methods. Proceedings of Mobile HCI'03, Italy. Lecture Notes in Computer Science, Springer-Verlag

8. Kjeldskov J. (2002) Just-In-Place Information for Mobile Device Interfaces. Proceedings of Mobile HCI'02, Italy, Lecture Notes in Computer Science, Springer-Verlag 
9. Pedell S., Graham C., Kjeldskov J. and Davies J. (2003) Mobile Evaluation: What the Metadata and the data told us. Proceedings of OzCHI 2003, Brisbane, Australia

10. Rogers Y., Scaife M., Harris E., et al. (2002) Things aren't what they seem to be: innovation through technology inspiration. Proceedings of DIS'02, London, ACM

11. Sato S. and Salvador T. (1999) Methods \& tools: Playacting and focus troupes: theater techniques for creating quick, intense, immersive, and engaging focus group sessions. Interactions of the ACM, 6 (5), pp 35-41

12. Vygotsky L. (1978) Mind in Society. MIT Press, Cambridge, MA 